\title{
Prevalence of Hypovitaminosis D in Patients Visiting a Tertiary Care Center in Chitwan, Nepal
}

\author{
Kushal Bhattarai, ${ }^{1}$ Nilu Manandhar, ${ }^{2}$ Sarita Dhakal ${ }^{1}$ \\ ${ }^{1}$ Department of Biochemistry, ${ }^{2}$ Department of Physiology, College of Medical Sciences and Teaching Hospital, \\ Bharatpur Chitwan, Nepal.
}

\begin{abstract}
Background: As documented by a large body of evidences, Hypovitaminosis D prevails both in the general and the hospitalized populations. We conducted this study to determine the prevalence of hypovitaminosis D amongst the patients visiting a tertiary care hospital and compare the rate gender-wise, across different categories of socio-demographic variables. Methods: It was a cross-sectional study (retrospective chart review) based on the hospital registry of patients at College of Medical Sciences and Teaching Hospital, Bharatpur, Chitwan, Nepal. From the registry, we retrieved the socio-demographic variables (age, gender, ethnicity and season) along with serum vitamin D (25-hydroxy-cholecalciferol) concentrations of the study subjects from January 2015 to August 2018, for our analysis. Hypovitaminosis D was defined as serum vitamin D $<30 \mathrm{ng} / \mathrm{mL}$ (insufficiency: 20-30ng/mL and deficiency: $<20 \mathrm{ng} / \mathrm{mL}$ ). Results: The overall prevalence of hypovitaminosis D was 70.7\% (insufficiency: 35.9\% and deficiency: 34.8\%), 72.4\% (females), 64.2\% (males), 76.3\% (first age-quartile), 74.5\% (miscellaneous ethnic group), and 73.9\% (Spring). Females had higher prevalence rates of hypovitaminosis D (and lower median serum 25-hydroxy-cholecalciferol levels) as compared to males, in overall participants, and those under each category of age, ethnicity and season (except Winter). Conclusions: The greater prevalence of hypovitaminosis D in the females than the males in the face of high rate in the overall hospital-visiting clearly calls for necessary strategies in the form of further studies and judicious prescription of supplementary vitamin D in the target population.
\end{abstract}

Keywords: hospital visiting patients; hypovitaminosis D; 25-hydroxy-cholecalciferol; vitamin D insufficiency; vitamin D deficiency.

\section{INTRODUCTION}

A lipid soluble vitamin and a prohormone, vitamin D orchestrates a plethora of physiological activities in the body apart from its most widely reported role in calcium homeostasis. ${ }^{1}$ The synthesis of this vitamin begins in the skin with further reactions taking place in the liver and the kidneys. ${ }^{2}$ An antecedent of this vitamin is cholecalciferol, which is synthesized in the skin by ultraviolet radiation (sunlight) mediated conversion from 7 dehydrocholesterol. Cholecalciferol undergoes two subsequent steps of hydroxylations to form calcidiol (25-hydroxycholecalciferol) in the liver and calcitriol (1,25-dihydroxycholecalciferol) in the kidneys. $^{3,4}$ Worldwide, hypovitaminosis $\mathrm{D}$ is reemerging as one of the chief community healthcare challenges, with over a billion people affected. ${ }^{5,6}$ The precedence this vitamin is receiving in the recent times can be attributed to the growing understanding of its pleotropic effects in the body such as metabolism, immunity, cardiovascular functions, growth and reproduction, to name a few. ${ }^{7,8}$ For the diagnosis of this clinical entity, clinicians rely on the biochemical findings of reduced levels of serum 25 hydroxycholecalciferol, supplemented by an array of meaningful clinical picture. Certain individuals in the general population are particularly predisposed to the shortage of this vitamin. Being a sunlight vitamin (as evident from its metabolism), any factors blocking the sunlight physically from skin such as brown/dark skin shade, pose a significant risk for hypovitaminosis D. ${ }^{9,10}$ Therefore, establishment of the levels of serum 25-hydroxy-cholecalciferol in total population and across different subgroups, such as gender, life-stage, ethnicity, seasonality is preemptive to the conceiving effectual stratagems to avert the inadequacy of this vitamin. ${ }^{11,12}$ Suboptimal concentrations of 25-hydroxycholecalciferol have been reported in as much as $50 \%$ of so-called healthy adult and elderly population. ${ }^{13}$ On top of this, several studies,

Correspondence: Dr. Kushal Bhattarai, Department of Biochemistry, College of Medical Sciences and Teaching Hospital, Bharatpur, Chitwan, Nepal. Email: kushal_2013@yahoo.com. Phone: +977-9804485207. Orcid ID: http:// orcid.org/0000-0002-8603-7060. DOI: 10.3126/jcmsn.v15i2.23483. Article received: 2019-01-04. Article accepted: 2019-05-08. 
conducted far and wide, report the overall prevalence of this condition from as low as $62.2 \%$ to as high as $87 \% .{ }^{14-19}$ To this end, the primary objective of our study was to establish the prevalence of hypovitaminosis D (including vitamin $D$ insufficiency and deficiency) in the patients visiting a tertiary healthcare center. We also aimed to compare the serum levels of vitamin D (25hydroxy-cholecalciferol) and the prevalence rates of hypovitaminosis $\mathrm{D}$ between two gender-groups (female and male) in the overall subjects as well as across different categories of demographic variables. As suggested by a plethora of studies conducted elsewhere, we anticipated lower levels of 25-hydroxy-cholecalciferol and thus, higher prevalence of hypovitaminosis $\mathrm{D}$ in the female subjects as compared to the males.

\section{METHODS}

This was a hospital-based analytical cross-sectional study, wherein, we performed a retrospective chart review (RCR) of patients visiting College of Medical Sciences and Teaching Hospital (COMS$\mathrm{TH})$. After obtaining ethical clearance from the Institutional Review Committee of COMS-TH (COMSTH-IRC), we retrieved the secondary data from the registry of Central Clinical Laboratory of the hospital. COMS-TH is a multi-specialty and super-specialty, tertiary care hospital that caters to a substantial share of healthcare needs of the residents in State-3 (Chitwan district) of Nepal. The data obtained comprised of the socio-demographic variables along with serum vitamin D (25-hydroxycholecalciferol) concentrations of all the individuals who had their blood investigated for serum vitamin D (25-hydroxy-cholecalciferol) in the COMS-TH Central Clinical Laboratory from January 2015 to August 2018 (44 months). After excluding the samples with serum vitamin D levels beyond (greater/less than) the detection limits of the instrument, and including only a single measurement for each participant during a particular single visit, a total sample consisting of 2617 participants was considered for the analysis. In these participants, the variables representing the demographic profile were (a) self-reported age in years (continuous variable), (b) gender (male/ female) (categorical variable), (c) ethnicity (based on self-reported surnames of the participants) (categorical variable), and (d) month of the year in which the vitamin D was measured in the laboratory (categorical variable). The variable "age" was discretized into different age-quartiles. Based on the self-reported surnames, the subjects were categorized into three ethnic groups, as (a) Indo-
Aryans (b) Tibeto-Burmans/Newars and (c) Miscellaneous. Lastly, the months of the year were further categorized into four seasons, as (a) Spring (March, April and May), (b) Summer (June, July, August), (c) Autumn (September, October and November), and (d) Winter (December, January and February). Serum vitamin D (25-hydroxycholecalciferol) was measured in Clinical Chemistry section of the Central Clinical Laboratory as one of the special biochemical tests, using Maglumi-2000 Biochemical Analyzer, with an in vitro competitive CLIA (Chemiluminescence Immunoassay) kit. As depicted in (Table 1), we categorized serum vitamin D concentrations of the study subjects into different categories as an approach to reflect its status.

\begin{tabular}{|c|c|}
\hline \multicolumn{2}{|c|}{$\begin{array}{l}\text { Table 1. Definition of vitamin D status according to serum } \\
\text { 25-hydroxy-cholecalciferol concentration. }\end{array}$} \\
\hline Vitamin D Status & $\begin{array}{l}\text { Serum 25-hydroxy- } \\
\text { cholecalciferol concentration }\end{array}$ \\
\hline 1. Hypervitaminosis D & $\geq 100 \mathrm{ng} / \mathrm{mL}$ \\
\hline 2. Normal Vitamin D & $\geq 30 \mathrm{ng} / \mathrm{mL}$ and $<100 \mathrm{ng} / \mathrm{mL}$ \\
\hline 3. Hypovitaminosis D & $<30 \mathrm{ng} / \mathrm{mL}$ \\
\hline 3.1. Vitamin D Insufficiency & $\geq 20 \mathrm{ng} / \mathrm{mL}$ and $<30 \mathrm{ng} / \mathrm{mL}$ \\
\hline 3.2. Vitamin D Deficiency & $<20 \mathrm{ng} / \mathrm{mL}$ \\
\hline
\end{tabular}

\section{Statistical Analysis}

From the registry of central clinical laboratory, raw data with respect to the variables of interest were retrieved in the form of Microsoft Excel 2007 documents for individual month (from January 2015 to August 2018). After the preliminary data management, we entered the data into SPSS (Statistical Package for Social Sciences) version 16.0 for analysis. As a descriptive statistical approach, we presented of the results in the form of appropriate tables, using frequency (with percentage) for categorical variables and measures of central tendency (mean with standard deviation and median with interquartile interval) for continuous variables. We tested the continuous variable, i.e., serum vitamin $\mathrm{D}(\mathrm{ng} / \mathrm{mL})$ for normality for overall and each category of the gender, age, ethnicity and season using graphs and appropriate statistical test. With the result showing the distributions to be significantly deviated from normality, we compared serum levels of 25hydroxy-cholecalciferol between females and males in each of these categories using nonparametric Mann-Whitney Test. Similarly, the prevalence rates of hypovitaminosis $\mathrm{D}$ were compared between females and males in overall patients and across the various categories of age, ethnicity and season, and the results were reported 
as absolute differences in proportions, and odds ratios (OR) (unadjusted and adjusted) with 95\% confidence intervals (CI). Adjusted OR was determined using binary logistic regression model, with the necessary adjustments done for various covariates, i.e., age, ethnicity and season, as necessary. Statistical significance was set at $\mathrm{p}$-value less than 0.05 .

\section{RESULTS}

We summarized the status of serum vitamin D (25hydroxy-cholecalciferol) across the different sociodemographic variables of a total 2617 subjects. We also compared the serum levels of this vitamin and prevalence rates of hypovitaminosis $\mathrm{D}$ between the two gender groups i.e., female and male in overall participants and in different subgroups of the other socio-demographic variables, i.e., age, ethnicity and season of the year. The study subjects included in the analysis were 3-95 years old with the mean (standard deviation) and median (interquartile range) ages of $47.2 \pm 16.9$ years and $46.0(35.0-60.0)$ years respectively. Females comprised of $78.8 \%$ $(n=2061)$ of the overall patients and were between 3-95 years old with the mean and median ages of $45.7 \pm 16.1$ years and $45.0(34.0-57.0)$ years, respectively. Similarly, males were between 7-94 years old with the mean and median ages of $52.9 \pm 18.5$ years and 54.5(38.0-68.0) years, respectively. The mean and median serum 25hydroxy-cholecalciferol concentrations in the overall patients were respectively, $26.5 \pm 13.9 \mathrm{ng} /$
$\mathrm{mL}$ and 23.3(17.9-31.6) $\mathrm{ng} / \mathrm{mL}$. According to the criteria defined in table 1 , serum vitamin $D$ of these patients were categorized and presented in (Table 2 ). The prevalence of hypovitaminosis $\mathrm{D}$ in the overall subjects was $70.7 \%(n=1850)$. Similarly, the proportions of the patients with insufficiency and deficiency of this vitamin were $35.9 \%(\mathrm{n}=940)$ and $34.8 \% \quad(n=910), \quad$ respectively. Gender-wise comparison of the vitamin D status showed fairly similar proportions of patients with insufficiency and deficiency in the female patients $(36.1 \%$ vs $36.3 \%$ ), with appreciable difference in the males $(35.1 \%$ vs $29.1 \%)$. Conversely, the prevalence rates of insufficiency didn't differ much between the two gender groups, even though females had a remarkably higher prevalence of deficiency as compared to males. The overall prevalence of hypovitaminosis $\mathrm{D}$ was higher in the females than in the males (72.4\% vs $64.2 \%)$. (Table 1 ; Table 2 ) Across the different age quartiles, we found that the prevalence of hypovitaminosis $\mathrm{D}$ was the highest in patients with the lowest quartile of age $(76.4 \%)$, and was the lowest in those with the highest agequartile $(61.5 \%)$. Further, the prevalence rates of deficiency were greater than insufficiency in the patients with the lowest (first) and second agequartiles $(41.7 \%$ vs $34.7 \%$ and $38.5 \%$ vs $36.1 \%)$. However, the trend reversed in the subjects with third and the highest (fourth) quartiles; with the deficiency rate lower than insufficiency $(32.0 \%$ vs $38.4 \%$ and $26.9 \%$ vs $34.6 \%$ ). Succinctly, prevalence of deficiency displayed a decreasing

Table 2. Status of serum 25-hydroxy-cholecalciferol (vitamin D) in the overall study subjects and according to different subgroups of gender, age quartile, ethnicity and season.

\begin{tabular}{|c|c|c|c|c|c|}
\hline \multirow[b]{3}{*}{ Overall } & \multicolumn{5}{|c|}{ Serum 25-Hydroxy-Cholecalciferol (vitamin D) Status } \\
\hline & Hypervitaminosis & Normal & Insufficiency & Deficiency & Total \\
\hline & $15(0.6 \%)$ & $752(28.7 \%)$ & $940(35.9 \%)$ & $910(34.8 \%)$ & 2617 \\
\hline \multicolumn{6}{|l|}{ Gender } \\
\hline Female & $10(0.5 \%)$ & $55(27.1 \%)$ & $745(36.1 \%)$ & $748(36.3 \%)$ & 2061 \\
\hline Male & $5(0.9 \%)$ & $19(34.9 \%)$ & $195(35.1 \%)$ & $162(29.1 \%)$ & 556 \\
\hline \multicolumn{6}{|l|}{ Age Quartiles } \\
\hline First & $2(0.3 \%)$ & $153(23.4 \%)$ & $227(34.7 \%)$ & $273(41.7 \%)$ & 655 \\
\hline Second & $2(0.3 \%)$ & $164(25.1 \%)$ & $236(36.1 \%)$ & $252(38.5 \%)$ & 654 \\
\hline Third & $5(0.8 \%)$ & $189(28.9 \%)$ & $251(38.4 \%)$ & $209(32.0 \%)$ & 654 \\
\hline Fourth & $6(0.9 \%)$ & $246(37.6 \%)$ & $226(34.6 \%)$ & $176(26.9 \%)$ & 654 \\
\hline \multicolumn{6}{|l|}{ Ethnic Groups } \\
\hline Indo-Aryan & $11(0.7 \%)$ & $460(29.0 \%)$ & $552(34.8 \%)$ & $564(35.5 \%)$ & 1587 \\
\hline Tibeto-Burman/Newar & $1(0.2 \%)$ & $18331.0 \%)$ & $230(38.9 \%)$ & $177(29.9 \%)$ & 591 \\
\hline Miscellaneous & $3(0.7 \%)$ & $109(24.8 \%)$ & $158(36.0 \%)$ & $169(38.5 \%)$ & 439 \\
\hline \multicolumn{6}{|l|}{ Seasons } \\
\hline Spring & $4(0.5 \%)$ & $228(25.7 \%)$ & $350(39.4 \%)$ & $306(34.5 \%)$ & 888 \\
\hline Summer & $5(0.6 \%)$ & $223(28.4 \%)$ & $281(35.8 \%)$ & $275(35.1 \%)$ & 784 \\
\hline Autumn & $4(1.1 \%)$ & $147(40.1 \%)$ & $134(36.5 \%)$ & $82(22.3 \%)$ & 367 \\
\hline Winter & $2(0.3 \%)$ & $154(26.6 \%)$ & $175(30.3 \%)$ & $247(42.7 \%)$ & 578 \\
\hline
\end{tabular}


trend with increased age-quartiles; whereas, the rate of insufficiency exhibited an increasing trend from the lowest to the third quartiles. The highest quartile, however, exhibited the lowest prevalence rate, for both insufficiency and deficiency. (Table 1; Table 2). Amongst the various ethnicities, the miscellaneous ethnic group had the highest prevalence of hypovitaminosis D (74.5\%), and Tibeto-Burman/Newar had the lowest (68.9\%). Likewise, the rates of deficiency were higher than the insufficiency in the Indo-Aryan and miscellaneous groups $(35.5 \%$ vs $34.8 \%$ and $38.5 \%$ vs $36.0 \%$ ). Notwithstanding, in the Tibeto-Burman/ Newar group, prevalence of deficiency was considerably lower than the rate of insufficiency $(29.9 \%$ vs $38.9 \%)$. In general, miscellaneous ethnic group had the highest prevalence of deficiency and Tibeto-Burman/Newar group had the lowest. As for the insufficiency state, Tibeto-Burman/Newar group had the highest rate and the Indo-Aryan group had the lowest. (Table 1; Table 2). Seasonal variation of the vitamin D status revealed the highest prevalence of hypovitaminosis D during Spring (73.9\%) and Winter $(73.0 \%)$ and the lowest rate in Autumn $(58.8 \%)$. Further scrutiny showed the rates of deficiency in Spring, Summer and Autumn to be lesser as compared to the insufficiency rates $(34.5 \%$ vs $39.4 \% ; 35.1 \%$ vs $35.8 \%$ and $22.3 \%$ vs $36.5 \%$ ). Winter, on the other hand, was characterized by a notably higher deficiency rate $(42.7 \%$ vs $30.3 \%)$. In summary, the rate of insufficiency was the highest in Spring and the lowest in Winter; whereas, the rate of deficiency was the highest in Winter and the lowest in Autumn. (Table 1; Table 2). We also compared the prevalence rates of hypovitaminosis $\mathrm{D}$ between male and female subjects in overall patients and in patients under each category of age quartile, ethnicity and season. We presented the results using absolute difference in proportion and odds ratio (OR) (unadjusted and adjusted). We employed a binary logistic regression model with different covariates used for necessary adjustments to determine the adjusted OR. The extent/degree of association and margins of uncertainty were expressed as of $95 \%$ confidence intervals (CI) of each statistical measure. Table 3 depicts the results of these comparison. As illustrated, females had higher prevalence rates of hypovitaminosis D as compared to males in the overall participants and in each of the categories of age, ethnicity and season (except Winter). Overall, females had 8.2\% greater prevalence rate of hypovitaminosis D as compared to males (95\% CI: $3.9 \%-12.7 \% ; \mathrm{p}<0.05$ ) and $36 \%$ greater odds of having the condition after adjusting for age, ethnicity and season (adjusted $\mathrm{OR}=1.36$; 95\%CI: $1.11-1.67 ; \mathrm{p}<0.05)$. Under each quartile of age, although the females had higher rates of hypovitaminosis $\mathrm{D}$ than the males, the differences

\begin{tabular}{|c|c|c|c|c|c|}
\hline \multicolumn{6}{|c|}{$\begin{array}{l}\text { Table 3. Comparison of the prevalence rates of hypovitaminosis D between male and female subjects in } \\
\text { overall participants and across different categories of age (age quartiles), ethnicity and season. }\end{array}$} \\
\hline & \multicolumn{2}{|c|}{ Hypovitaminosis D } & \multirow{3}{*}{$\begin{array}{l}\text { Difference in } \\
\text { Proportion } \\
(95 \% \text { CI) }\end{array}$} & \multicolumn{2}{|c|}{ Odds Ratio (OR) } \\
\hline & Female & Male & & Unadjusted & Adjusted* \\
\hline & $\mathbf{n}(\%)$ & n(\%) & & $(95 \% \mathrm{CI})$ & $(95 \% \mathrm{CI})$ \\
\hline Overall & $1493(72.40 \%)$ & $357(64.20 \%)$ & $8.2(3.9-12.7) \%$ & $1.47(1.20-1.79)$ & $1.36(1.11-1.67)$ \\
\hline \multicolumn{6}{|l|}{ Age Quartiles } \\
\hline First & $426(77.70 \%)$ & $74(69.20 \%)$ & $8.5(-0.3-18.4) \%$ & $1.56(0.99-2.46)$ & $1.59(1.00-2.52)$ \\
\hline Second & $421(75.40 \%)$ & $67(69.80 \%)$ & $5.6(-3.5-16.0) \%$ & $1.33(0.83-2.14)$ & $1.38(0.85-2.25)$ \\
\hline Third & $383(72 \%)$ & $77(63.10 \%)$ & $8.9(-0.1-18.5) \%$ & $1.5(0.99-2.27)$ & $1.49(0.98-2.26)$ \\
\hline Fourth & $263(62.60 \%)$ & $139(60.20 \%)$ & $2(-5.7-9.8) \%$ & $1.09(0.78-1.51)$ & $1.09(0.79-1.53)$ \\
\hline \multicolumn{6}{|l|}{ Ethnic Groups } \\
\hline Indo-Aryan & $917(72 \%)$ & $199(63.40 \%)$ & $8.6(2.9-14.6) \%$ & $1.49(1.15-1.93)$ & $1.33(1.02-1.75)$ \\
\hline \multicolumn{6}{|l|}{ Tibeto-Burman/ } \\
\hline Miscellaneous & $254(75.80 \%)$ & $73(70.20 \%)$ & $5.6(-3.7-15.9) \%$ & $1.33(0.82-2.17)$ & $1.34(0.81-2.18)$ \\
\hline \multicolumn{6}{|l|}{ Seasons } \\
\hline Spring & $547(76.4 \%)$ & $109(63.4 \%)$ & $13(5.4-21.0) \%$ & $1.87(1.31-2.67)$ & $1.7(1.18-2.45)$ \\
\hline Summer & $428(73.7 \%)$ & $128(63.1 \%)$ & $10.6(3.3-18.2) \%$ & $1.64(1.17-2.30)$ & $1.54(1.09-2.17)$ \\
\hline Autumn & $178(60.8 \%)$ & $38(51.4 \%)$ & $9.4(-3.0-21.8) \%$ & $1.47(0.88-2.45)$ & $1.41(0.84-2.38)$ \\
\hline Winter & $340(72.2 \%)$ & $82(76.6 \%)$ & $4.4(-12.6-5.3) \%$ & $0.79(0.48-1.29)$ & $0.7(0.42-1.17)$ \\
\hline \multicolumn{6}{|c|}{ *OR, adjusted for: } \\
\hline \multicolumn{6}{|c|}{ a) Age, Ethnicity and Seasons (in overall participants) } \\
\hline \multicolumn{6}{|c|}{ b) Ethnicity and Seasons (in each age quartile) } \\
\hline \multicolumn{6}{|c|}{ c) Age and Seasons (in each ethnic group) } \\
\hline d) Age and Et & ity (in each seas & & & & \\
\hline
\end{tabular}


Bhattarai et al. Prevalence of Hypovitaminosis D in Patients Visiting a Tertiary Care..

were not statistically significant $(\mathrm{p}>0.05)$. Likewise, across the different ethnic groups, IndoAryan females had $8.6 \%$ higher prevalence rates of hypovitaminosis $\mathrm{D}$ than the respective males (95\% CI: $2.9 \%-14.6 \% ; \mathrm{p}<0.05)$ and $33 \%$ greater odds (adjusted OR: 1.33; 95\% CI: 1.02-1.75; $\mathrm{p}<0.05)$. Although Tibeto-Burman and Newar females had $9.5 \%$ higher rate of the condition than the males $(95 \% \mathrm{CI}: 0.7 \%-18.7 \% ; \mathrm{p}<0.05)$, the adjusted OR was 1.44 (95\% CI: 0.95-2.18; $\mathrm{p}>0.05)$. For miscellaneous group, the gender-wise difference in the rate was not statistically significant $(p>0.05)$. Amongst the various seasons, females who had their vitamin D assessed in Spring had $13.0 \%$ greater prevalence of hypovitaminosis D as compared to the males $(95 \%$ CI: $5.4 \%-21.0 \% ; \mathrm{p}<0.05)$ and $70 \%$ greater odds (adjusted OR=1.70; 95\% CI: 1.18-2.45; $\mathrm{p}<0.05$ ). Similarly, in Summer, the difference in proportion, i.e., $10.6 \%(95 \%$ CI: $3.3 \%-18.2 \%$; p $<0.05)$ was significant (adjusted $\mathrm{OR}=1.54$; 95\% CI: 1.09$2.17 ; \mathrm{p}<0.05)$. The gender-wise differences in ethnicity and those who had their vitamin D assessed in Spring, Summer and Autumn seasons $(\mathrm{p}<0.05)$.

\section{DISCUSSION}

A growing body of evidence reporting a high prevalence rate of hypovitaminosis D in the general population and the hospital-visiting patients, prompted the present study. As reported in our study, the overall prevalence of hypovitaminosis D was $70.7 \%$. Several studies conducted in different regions of the world have reported similar findings. In one study based on Indian children and adolescents, Vasudevan ${ }^{14}$ established the prevalence rate of hypovitaminosis $\mathrm{D}$ as $62.2 \%$. Mansour et al, ${ }^{15}$ in their study settled with $72.6 \%$ as the overall prevalence rate of this condition. Similarly, Shrestha et al ascertained an overall prevalence rate of $78.2 \%$ in the Nepali youths of Lalitpur. ${ }^{16}$ In an Australian study carried out on the East African migrants, the prevalence of hypovitaminosis D was $87 \%{ }^{19}$ As reported by

Table 4. Comparison of median (interquartile range, IQR) serum 25-hydroxy-cholecalciferol levels between female and male patients in overall participants and across different categories of age (age quartiles), ethnicity and season.

\begin{tabular}{|c|c|c|c|c|c|c|}
\hline & \multicolumn{4}{|c|}{ Serum 25-Hydroxy-Cholecalciferol (ng/mL) } & \multicolumn{2}{|c|}{ (Mann-Whitney Test) } \\
\hline & \multicolumn{2}{|c|}{ Female } & \multicolumn{2}{|c|}{ Male } & \multirow[b]{2}{*}{$\mathbf{Z}$} & \multirow[b]{2}{*}{ P-value } \\
\hline & Median & IQR & Median & IQR & & \\
\hline Overall & 22.9 & $17.7-31.0$ & 28.9 & $18.9-34.1$ & -4.04 & $<0.001$ \\
\hline \multicolumn{7}{|l|}{ Age Quartiles } \\
\hline First & 21.8 & $16.9-29.1$ & 23.4 & $17.5-33.2$ & -1.61 & 0.107 \\
\hline Second & 22.4 & $17.5-29.8$ & 23.5 & $18.2-33.1$ & -1.21 & 0.227 \\
\hline Third & 22.7 & $18.3-31.1$ & 26 & $19.1-33.0$ & -2.25 & 0.025 \\
\hline Fourth & 26 & $19.3-33.7$ & 26 & $19.8-36.1$ & -0.77 & 0.441 \\
\hline \multicolumn{7}{|l|}{ Ethnic Groups } \\
\hline Indo-Aryan & 22.9 & $17.7-31.0$ & 24.7 & $18.6-33.3$ & -2.68 & 0.007 \\
\hline Tibeto-Burman/Newar & 23.8 & $18.5-31.5$ & 26.4 & $20.3-35.3$ & -2.89 & 0.004 \\
\hline Miscellaneous & 21.9 & $17.0-29.5$ & 23.7 & $17.1-35.0$ & -1.22 & 0.221 \\
\hline \multicolumn{7}{|l|}{ Seasons } \\
\hline Spring & 22.7 & $17.8-29.3$ & 25.3 & $19.6-33.4$ & -3.39 & 0.001 \\
\hline Summer & 22.8 & $17.6-30.4$ & 25.2 & $18.9-34.5$ & -3.02 & 0.002 \\
\hline Autumn & 26.5 & $20.2-34.7$ & 29.9 & $21.4-45.7$ & -2.34 & 0.02 \\
\hline Winter & 21.5 & $16.1-31.6$ & 21.3 & $15.9-29.2$ & 0.6 & 0.546 \\
\hline
\end{tabular}

prevalence rates were not significant in Autumn and Winter $(\mathrm{p}>0.05)$ (Table 1; Table 4).

As depicted in Table 4, median serum vitamin D (25-hydroxy-cholecalciferol) concentration was less in female as compared to males in overall participants and those under each categories of age, ethnicity and season (except Winter). The differences were statistically significant only in overall subjects, subjects in the third age-quartile, with Indo-Aryan and Tibeto-Burman/Newar
Gannage-Yared et $\mathrm{al}^{17}$, the prevalence of this condition in the general population was $72.8 \%$. Bhatta et $\mathrm{al}^{18}$ evaluated vitamin D status from the serum values of 25-hydroxycholecalciferol measured in a clinical laboratory in Western Nepal. In a total sample of 2158 patients $19-60$ years old, they reported the overall prevalence of hypovitaminosis $\mathrm{D}$ to be $73.7 \%$. Regmi et $\mathrm{al}^{20}$, in their study based on the children and young adult population visiting another tertiary healthcare 
center in Chitwan, reported the prevalence of hypovitaminosis D to be $74.1 \%$. Studies have suggested upto $70 \%$ of adolescents to be suffering from hypovitaminosis $\mathrm{D}$, with the proportions even more in younger subjects. ${ }^{21,22}$ These findings are in accordance with our study, where the prevalence of the condition in the first quartile of age was $76.4 \%$.

Further, our study has reported the prevalence of hypovitaminosis $\mathrm{D}$ to be the highest in the lowest quartile and the lowest in the highest quartile of age $(61.5 \%)$. This observed pattern of increased prevalence with decreased age can be attributed to various factors. An important one, also reiterated in the study by Regmi et $\mathrm{al}^{20}{ }^{20}$ could be an inclusion of more proportions of afflicted children, who are naturally more likely to be taken to the hospital by their parents than any other age-groups on account of any relevant clinical picture, whatsoever. Amongst the children, specifically, those reliant solely on mothers' milk are superfluously at risk. Notwithstanding, when the mother has hypovitaminosis D and/or gives birth to premature babies, the infants are highly likely to suffer from this nutritional imbalance. ${ }^{9}$ Although we observed the lowest prevalence of hypovitaminosis in the highest age-quartile, proportions of insufficient subjects did not differ much between the different age-quartiles. Naturally, manufacture of this vitamin D in the skin has been reported to tumble with age. ${ }^{23}$

Amidst the various likely contributors of hypovitaminosis D, some of the most potential ones are contracted exposure to sunbeams as a result of forsaken outdoor activities, no matter whether recreational or professional. ${ }^{20}$ In addition, unclean and contaminated atmosphere as a result of increased urbanization is also equally accountable. ${ }^{24}$ Polluted air particles physically block the UV irradiation from reaching the skin. ${ }^{25}$ To reiterate, urban citizens spend most of their time indoors, resulting in diminished sunlight exposure. ${ }^{26}$ As per our findings, the prevalence of hypovitaminosis $\mathrm{D}$ was higher in the females $(72.4 \%)$ as compared to the males $(64.2 \%)$, and the difference in proportion was statistically significant $(p<0.05)$. Likewise, females had significantly greater odds of having this condition, after adjusting for age, ethnicity and seasonal variations $(O R=1.36 ; \mathrm{p}<0.05)$. Besides, they had a significantly lower median serum vitamin $D$ levels as compared to the males $(p<0.0 .5)$. In accord to these outcomes, it is meaningful to state that many studies too have reported a higher prevalence of hypovitaminosis D in females. Bhatta et $\mathrm{al}^{18}$, found the prevalence of hypovitaminosis $\mathrm{D}$ to be $75.4 \%$ in females and $70.1 \%$ in males. Regmi et $\mathrm{al}^{20}$ reported a higher prevalence of hypovitaminosis $\mathrm{D}$ in females (children and youngsters) $(80.9 \%)$ as compared to males $(68.9 \%)$ of same age-group. Although the authors didn't report any statistical inference, the difference in proportion (i.e., 12.0\%) was in contrast to our finding, especially in the first quartile of age. Other studies reporting higher prevalence of hypovitaminosis D in the female population are those by Verdoia et $\mathrm{al}^{27}$; GannageYared et $\mathrm{al}^{17}$; and Bolland et $\mathrm{al}^{28}$. Of the various factors accounting for such differences, societal norms culminating in gender-wise variances in length of sunlight-exposure could be the most substantial. These could be better epitomized in certain ethnic groups, in which women not only spend much of their time indoors, but also cover their bodies even when they are outdoors. ${ }^{24}$ Furthermore, the characteristic distribution of fats in females is one important reason for the lower serum levels of vitamin D in this gender group, as more vitamin $\mathrm{D}$ gets sequestered into the adipose tissues. $^{29}$

In our study, for each age-quartile, though the females had a higher prevalence of hypovitaminosis $\mathrm{D}$ than the males, we couldn't find any statistically significant difference $(p>0.05)$. Similarly, the median vitamin D levels didn't differ significantly between males and females across the different agequartiles, except for the third quartile where females had significantly lower levels than males $(p=0.025)$. Amongst the different ethnic groups, patients with miscellaneous ethnicity had the highest prevalence of hypovitaminosis D (74.5\%) with the Tibeto-Burman and Newar ethnicity having the lowest $(68.9 \%)$. Bhatta et $\mathrm{al}^{18}$ reported the Brahmins to be having the highest rate as compared to the other ethnicity. In our study, gender-wise comparison revealed the females to have greater prevalence in all ethnic groups; however, only the Indo-Aryan females had significantly higher adjusted odds of hypovitaminosis $\mathrm{D}$ as compared to the males $(\mathrm{OR}=1.33 ; \mathrm{p}<0.05)$. However, the both the IndoAryan and Tibeto-Burman/Newar females had a significantly lower median vitamin $\mathrm{D}$ as compared to the respective males $(p<0.05)$. Assessment of the relationship of the vitamin $\mathrm{D}$ status with different seasons and demonstration of season-wise fluctuation of hypovitaminosis $\mathrm{D}$ have been the norms of many studies, such as those by van der 
Mei et $\mathrm{al},{ }^{30}$ Goswami et al, ${ }^{31}$ Daly et $\mathrm{al},{ }^{32}$ and Choi, ${ }^{33}$ etc. According to Bhatta et $\mathrm{al}^{18}$, patients who had their serum vitamin $\mathrm{D}$ measured in the Spring had the highest prevalence of hypovitaminosis $\mathrm{D}(88.8 \%)$. The rate was the lowest in the Autumn (57.1\%). In our study, the prevalence of hypovitaminosis $\mathrm{D}$ was the highest in the Spring and Winter $(73.9 \% ; 73.0 \%)$ and the lowest in the Autumn (58.8\%). Females had greater prevalence rates than males in all seasons, except for the Winter (the males had higher prevalence). The gender-wise differences in proportions were statistically significant in Spring and Summer seasons $(\mathrm{p}<0.05)$. Moreover, in Spring, females had significantly greater adjusted odds of having hypovitaminosis D than males $(\mathrm{OR}=1.70 ; \mathrm{p}<0.05)$; in Summer, the adjusted OR of similar comparison was $1.54(\mathrm{p}<0.05)$. The median serum vitamin D levels were significantly higher in the males than in the females in Spring, Summer and Autumn $(p<0.05)$ and not in the Winter $(\mathrm{p}>0.05)$.

We employed the cutoff values of $30 \mathrm{ng} / \mathrm{mL}$ and 20 $\mathrm{ng} / \mathrm{mL}$ for serum 25-hydroxy-cholecalciferol to define insufficiency and deficiency of vitamin D, respectively. In agreement, most experts have also used the similar cutoff values. ${ }^{5}$ However, many other experts consider $10 \mathrm{ng} / \mathrm{mL}$ and $20 \mathrm{ng} / \mathrm{mL}$ as the cutoff points to define deficiency and insufficiency of this vitamin, respectively. ${ }^{34,35}$ Many studies report the prevalence rates of vitamin D insufficiency and deficiency to vary from $52-77 \%$, and $18-36 \%$ respectively. ${ }^{32}$ In our study, the overall prevalence rates of vitamin D insufficiency and deficiency were $35.9 \%$ and $34.8 \%$ respectively. In the study by Regmi et al, ${ }^{20}$ $17.6 \%$ of the study subjects were insufficient; $56.5 \%$ were deficient; and $27.8 \%$ were severely deficient (levels $<10 \mathrm{ng} / \mathrm{mL}$ ) in vitamin D. The study population in that study were children and youngsters, i.e., the age-group comparable to the first quartile of age in our study. We found in our study that patients in the first and second quartiles of age demonstrated greater proportions of deficiency, whereas those in the third and last quartiles showed greater proportions of insufficiency. To this end, in a cross-sectional study based on 500 random pairs of mother and infant conducted in Nepal, Haugen et $\mathrm{al}^{36}$ found that $3.6 \%$ of infants and $59.8 \%$ of mothers had serum vitamin D levels $<20 \mathrm{ng} / \mathrm{mL}$. In another study, Cashman et al, ${ }^{37}$ based on the criteria of VDSP (Vitamin D Standardization Program) as put into operation in 14 studies, found the pooled prevalence of vitamin D levels less than $30 \mathrm{nmol} / \mathrm{L}$ $(\sim 12 \mathrm{ng} / \mathrm{mL})$ to be $13.0 \%$ amongst the Europeans. Likewise, using cutoff of $50 \mathrm{nmol} / \mathrm{L}$, the prevalence was $40.4 \%$. In our study we found that the rates of deficiency were higher than the insufficiency in the Indo-Aryan and miscellaneous groups. Notwithstanding, in the Tibeto-Burman/Newar group, prevalence of deficiency was considerably lower than the rate of insufficiency. Likewise, season-wise comparisons showed the prevalence rates of deficiency to be lesser than those of insufficiency in Spring, Summer and Autumn; whereas, deficiency rate higher than that of insufficiency was reported in Winter. Cashman et $\mathrm{al}^{37}$ also observed the prevalence of deficiency to decrease during summer and increase during Winter. Similarly, in regard to the ethnicity, they reported the dark-skinned individuals to be suffering from deficiency more frequently as compared to the light-skinned ones.

The limitations incumbent in the present study can be aptly attributed to the study design. It was a hospital-based study with the data collected from the registry by retrospective chart review (RCR) and thus doesn't represent the general population. The vitamin D status as described in the present study were specifically those of the patients who visited the hospital with some clinical problems and got their serum vitamin D investigated mostly after their physicians ordered for the tests. This clearly points towards the lack of generalizability of the findings to the general population. Nonetheless, the outcomes of the present study can be used as references in future if some related researches are to be conducted at the same institution. Secondly, in our study, we couldn't report factors other than age, gender, ethnicity and seasons to account for their possible effects on vitamin D status in the hospitalvisiting population. Third, reporting of vitamin $\mathrm{D}$ status alone in the face of paucity of overall clinical status (such as comorbidities, medications, other laboratory parameters such as calcium, phosphate, etc) of the participants clearly points towards the fallibility in depicting a far-reaching picture. Serum levels of calcium, phosphorus, parathyroid hormones, etc have been found to be meaningfully associated with serum 25-hydroxy-cholecalciferol concentrations. ${ }^{4}$

\section{CONCLUSIONS}

The overall prevalence of hypovitaminosis D was $70.7 \%$ (insufficiency: $35.9 \%$ and deficiency: $34.8 \%)$. It was greater in the females than in the males in overall participants as well as those under 
each category of age, ethnicity and season (except Winter). Moreover, the rate was the greatest in the first quartile of age, miscellaneous ethnic groups and during the Spring season. Based on these findings and the critical analyses of our shortcomings, we recommend that further epidemiological surveys should be conducted to highlight other important factors associated with hypovitaminosis D. As a matter of fact, subsequent longitudinal studies are also central to clearly account for the causative roles of these factors. Lastly, judicious incorporation of vitamin-
$\mathrm{D}$ in the diet is as important as ample and proper exposure to the sunlight.

\section{ACKNOWLEDGEMENTS}

We are indebted to Mr. Prabodh Shrestha, Incharge, Central Clinical Laboratory, College of Medical Sciences and Teaching Hospital, for his valuable support in retrieval of the data form the hospital registry.

\section{Conflict of Interest: None}

T, Daida YG, Lensmeyer G, et al. Low vitamin D status despite abundant sun exposure. J Clin Endocrinol Metab. 2007;92(6):2130-5.

11.Sempos CT, Vesper HW, Phinney KW, Thienpont LM, Coates PM. Vitamin D status as an international issue: National surveys and the problem of standardization. Scand J Clin Lab Invest. 2012;243:32-40.

12.Cashman KD, Kiely M. Towards prevention of vitamin D deficiency and beyond: Knowledge gaps and research needs in vitamin D nutrition and public health. $\mathrm{Br} J$ Nutr. 2011;106 (11):1617-27.

13.Gordon CM, DePeter KC, Feldman HA, Grace E, Emans SJ. Prevalence of vitamin D deficiency among healthy adolescents. Arch Pediatr Adolesc Med. 2004;158(6):531-7.

14.Vasudevan J. prevalence and factors associated with vitamin $\mathrm{D}$ deficiency in indian children: a hospital based cross sectional study. Pediatr Oncall J. 2014;11(3).

15.Mansour MMHK, Alhadidi KM. Vitamin D deficiency in children living in Jeddah, Saudi Arabia. J Endocrinol Metab. 2012;16(2):263-9.

16.Shrestha S, Shrestha L, Jha DK. Vitamin D status among individuals attended in Health Home Care Centre, Lalitpur, Nepal. Nepal Med Coll J. 2012;14(2):84-7.

17.Gannagé-Yared MH, Chemali R, Yaacoub N, Halaby G. Hypovitaminosis D in a sunny country: Relation to lifestyle and bone markers. J Bone Miner Res. 2000;15(9):1856-62.

18. Bhatta MP, Pandey BR, Gurung KM, Nakarmi R, Gurung K, Gurung LB, et al. Prevalence of vitamin $\mathrm{D}$ deficiency among adult population of Western Region of Nepal. Int J Med Biomed Sci. 2016;1(2):7-12.

19.McGillivray G, Skull SA, Davie G, Kofoed SE, Frydenberg A, Rice J, et al. High prevalence of asymptomatic vitamin D and iron deficiency in East African immigrant children and adolescents living in a temperate climate. Arch Dis Child. 2007;92(12):1088-93. 
20.Regmi S, Regmi AP, Adhikari S, Shakya D. Prevalence of vitamin D deficiency/ insufficiency among children and adolescents. J Chitwan Med Coll. 2017;7(19):11-5.

21.Moussavi M, Heidarpour R, Aminorroaya A, Pournaghshband Z, Amini M. Prevalence of vitamin D deficiency in Isfahani high school students in 2004. Horm Res. 2005;64(3):144 8.

22.Hovsepian S, Amini M, Aminorroaya A, Amini P, Iraj B. Prevalence of Vitamin D deficiency among adult population of Isfahan city, Iran. J Heal Popul Nutr. 2011;29(2):14955.

23.Holick MF, Matsuoka LY, Wortsman J. Age, vitamin $\mathrm{D}$, and solar ultraviolet. Lancet. 1989;2(8671):1104-5.

24.Sachan A, Gupta R, Das V, Agarwal A, Awasthi PK, Bhatia V. High prevalence of vitamin $\mathrm{D}$ deficiency among pregnant women and their newborns in northern India. Am J Clin Nutr. 2005;81(5):1060-4.

25.Hosseinpanah F, Pour SH, Heibatollahi M, Moghbel N, Asefzade S, Azizi F. The effects of air pollution on vitamin $\mathrm{D}$ status in healthy women: A cross sectional study. BMC Public Health. 2010;10:519.

26.Puri S, Marwaha RK, Agarwal N, Tandon N, Agarwal R, Grewal K, et al. Vitamin D status of apparently healthy schoolgirls from two different socioeconomic strata in Delhi: Relation to nutrition and lifestyle. Br J Nutr. 2008;99(4):876-82.

27.Verdoia M, Schaffer A, Barbieri L, Di Giovine G, Marino P, Suryapranata H, et al. Impact of gender difference on vitamin $\mathrm{D}$ status and its relationship with the extent of coronary artery disease. Nutr Metab Cardiovasc Dis. 2015;25 (5):464-70.

28.Bolland MJ, Grey AB, Ames RW, Horne AM, Mason BH, Wattie DJ, et al. Age-, gender-, and weight-related effects on levels of 25hydroxyvitamin $\mathrm{D}$ are not mediated by vitamin D binding protein. Clin Endocrinol. 2007;67 (2):259-64.
29.Dijkstra SH, Van Beek A, Janssen JW, De Vleeschouwer LHM, Huysman WA, Van Den Akker ELT. High prevalence of vitamin D deficiency in newborn infants of high-risk mothers. Arch Dis Child. 2007;92(9):750-3.

30.van der Mei IAF, Ponsonby AL, Engelsen O, Pasco JA, McGrath JJ, Eyles DW, et al. The high prevalence of vitamin D insufficiency across Australian populations is only partly explained by season and latitude. Environ Health Perspect. 2007;115(8):1132-9.

31.Goswami R, Gupta N, Goswami D, Marwaha RK, Tandon N, Kochupillai N. Prevalence and significance of low 25-hydroxyvitamin D concentrations in healthy subjects in Delhi. Am J Clin Nutr. 2000;72(2):472-5.

32.Daly RM, Gagnon C, Lu ZX, Magliano DJ, Dunstan DW, Sikaris KA, et al. Prevalence of vitamin $\mathrm{D}$ deficiency and its determinants in Australian adults aged 25 years and older: A national, population-based study. Clin Endocrinol (Oxf). 2012;77:26-35.

33.Choi EY. 25(OH)D status and demographic and lifestyle determinants of 25(OH)D among Korean adults. Asia Pac J Clin Nutr. 2012;21 (4):526-35.

34.IOM (Institute of Medicine). Dietary Reference Intakes for Calcium and Vitamin D. Washington. Washington, DC: The National Academies Press. 2011.

35.Gallagher JC, Sai AJ. Vitamin D insufficiency, deficiency, and bone health. J Clin Endocrinol Metab. 2010;95(6):2630-3.

36. Haugen J, Ulak M, Chandyo RK, Henjum S, Thorne-Lyman AL, Ueland PM, et al. Low Prevalence of Vitamin D Insufficiency among Nepalese Infants Despite High Prevalence of Vitamin D Insufficiency among Their Mothers. Nutrients. 2016 Dec 21;8(12):825.

37.Cashman KD, Dowling KG, Škrabáková Z, Gonzalez-Gross M, Valtueña J, De Henauw S, et al. Vitamin D deficiency in Europe: pandemic? Am J Clin Nutr. 2016 Apr 1;103 (4):1033-44.

Citation: Bhattarai K, Manandhar N, Dhakal S. Prevalence of Hypovitaminosis D in Patients Visiting a Tertiary Care Center in Chitwan, Nepal. JCMS Nepal. 2019; 15(2):84-92. 